\title{
Changes in histone gene dosage alter transcription in yeast
}

\author{
Chris D. Clark-Adams, ${ }^{1}$ David Norris, ${ }^{2}$ Mary Ann Osley, ${ }^{2,3}$ Jan S. Fassler,, ${ }^{1,4}$ and Fred Winston ${ }^{1}$ \\ ${ }^{1}$ Department of Genetics, Harvard Medical School, Boston, MA 02115 USA; ${ }^{2}$ Dana-Farber Cancer Center, Boston, MA 02115 \\ USA and Department of Microbiology and Molecular Genetics, Harvard Medical School, Boston, MA 02115 USA
}

\begin{abstract}
Chromatin structure is believed to be important for a number of cellular processes, including transcription. However, the role of nucleosomes in transcription is not well understood. We have identified the yeast histone locus HTB1-HTB1, encoding histones $\mathrm{H} 2 \mathrm{~A}$ and $\mathrm{H} 2 \mathrm{~B}$, as a suppressor of solo $\delta$ insertion mutations that inhibit adjacent gene expression. The HTA1-HTB1 locus causes suppression either when present on a high-copy-number plasmid or when mutant. These changes in HTA1-HTB1 alter transcription of the genes adjacent to the $\delta$ insertions. On the basis of this result, we have examined the effects of increased and decreased histone gene dosage for all four yeast histone loci. From the types of histone gene dosage changes that cause suppression of insertion mutations, we conclude that altered stoichiometry of histone dimer sets can alter transcription in yeast.
\end{abstract}

[Key Words: Histones; transcription; gene expression; yeast]

Received November 10, 1987; accepted December 31, 1987.

Many studies have demonstrated the presence of altered chromatin structure in transcriptionally active regions of the genome (for reviews, see Mathis et al. 1980; Pederson et al. 1986). However, other work has shown that the level of transcription does not affect chromatin structure at certain loci (Nasmyth 1982; for review, see Mathis et al. 1980). Thus, the cause-and-effect relationship between chromatin structure and transcription remains unclear.

The major subunit of chromatin is the nucleosome, which consists of DNA wrapped around a histone octamer that is composed of two H2A-H2B dimers and an H3-H4 tetramer (for review, see Pederson et al. 1986). Since histones are the protein components of nucleosomes, it is reasonable to hypothesize that alteration of histone levels would affect chromatin structure. A genetic approach to study the effect of chromatin structure on transcription and other cellular processes would be to alter histone levels by mutation or by gene amplification and then to screen for resulting mutant phenotypes.

Changes in histone levels have been reported to cause various phenotypes. Meeks-Wagner and Hartwell (1986) showed that a high copy number of the genes encoding $\mathrm{H} 2 \mathrm{~A}$ and $\mathrm{H} 2 \mathrm{~B}$ or of the genes encoding $\mathrm{H} 3$ and $\mathrm{H} 4$ causes increased chromosome loss during mitosis in Saccharomyces cerevisiae. Yeast cells depleted for $\mathrm{H} 2 \mathrm{~B}$ arrest in

\footnotetext{
${ }^{3}$ Present address: Program in Molecular Biology and Virology, Memorial Sloan-Kettering Cancer Center, 1275 York Avenue, New York, NY 10021 USA.

4Present address: Department of Biology, University of Iowa, Iowa City, Iowa 52242 USA.
}

mitosis, with defects in chromatin structure and nuclear segregation (Han et al. 1987). Norris and Osley (1987) demonstrated that deletion of one of the two H2A-H2B loci affects many aspects of the yeast life cycle, including the mitotic cell cycle and the stress response. In Drosophila melanogaster, a reduction of histone gene copy number suppresses gene inactivation associated with position-effect variegation (Moore et al. 1983). These studies suggest that altering histone levels can cause pleiotropic phenotypes, perhaps due to perturbations in chromatin structure.

If a wild-type level of histones is required for normal transcription, a genetic screen for transcription mutants might identify mutations in histone genes. We have isolated a large number of transcription mutants in the yeast Saccharomyces cerevisiae as suppressors of $\mathrm{Ty}$ and solo $\delta$ insertion mutations that inhibit normal transcription of adjacent genes (Winston et al. 1984b, 1987; Fassler and Winston 1988). Mutations in SPT genes (SPT $=$ suppressor of $\mathrm{Ty} /$ suppress $\mathrm{Ty}$ and $\delta$ insertion mutations by restoration of functional transcription to the adjacent genes (Silverman and Fink 1984; Winston et al. 1984a; Fassler and Winston 1988). Additionally, mutations in SPT genes confer various other mutant phenotypes, and some SPT genes are essential for growth, suggesting that they are required for more general aspects of cellular transcription (Winston et al. 1984a,b; Clark-Adams and Winston 1987; Fassler and Winston 1987).

Recently, we have shown that increased dosage of the wild-type SPT6 gene causes suppression of $\delta$ insertion 
mutations at the transcriptional level (Clark-Adams and Winston 1987; unpubl.). On the basis of this result, we have screened for other high-copy-number clones that can suppress $\delta$ insertion mutations. One of the highcopy-number clones isolated by this screen contains the genes previously identified as SPT11 and SPT12 (Fassler and Winston 1988). Genetic and molecular evidence demonstrates that these are the same genes as the histone genes HTA1 and HTB1. Analysis of altered gene dosage of this locus and of the other histone loci in yeast suggests that a change in the normal stoichiometry of histone proteins alters transcription in yeast.

\section{Results \\ Isolation of high-copy-number suppressors of $\delta$ insertion mutations}

Previous studies of the SPT6 gene demonstrated that increased dosage of the wild-type SPT6 gene, as well as mutations in SPT6, causes suppression of $\delta$ insertion mutations (Winston et al. 1984b; Clark-Adams and Winston 1987). Based on this result, we screened for other genes that in high copy number can suppress insertion mutations. We transformed a yeast library in the high-copy-number plasmid YEp24 (Carlson and Botstein

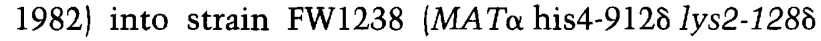
ura3-52). Ura ${ }^{+}$transformants were screened for suppression of the solo $\delta$ insertion mutation lys2-128 from Lys $^{-}$to Lys ${ }^{+}$. Thirty-one Lys ${ }^{+}$candidates were isolated from approximately $50,000 \mathrm{Ura}^{+}$transformants. Thirty of the candidates also suppressed his4-9128, resulting in a His ${ }^{+}$phenotype; the single $\mathrm{Lys}^{+} \mathrm{His}^{-}$clone contained the LYS2 gene as determined by restriction endonuclease mapping. The remaining clones fell into two classes by restriction endonuclease mapping and Southern hybridization analysis. Class 1 contained 17 members; class 2 contained 13 members. Our failure to reisolate the SPT6 gene from this particular screen probably reflects the large size of SPT6 (4.5 kb) (Clark-Adams and Winston 1987; Neigeborn et al. 1987), since the 4.2$\mathrm{kb}$ LYS2 gene was isolated only once.

To confirm that the suppression phenotype caused by the class 1 and class 2 clones was due to high copy number, we subcloned large restriction fragments from each class into the low-copy-number centromere-containing vector YCp50 (Johnston and Davis 1984) and into the high-copy-number vector YEp24. The low-copynumber subclones do not cause suppression of his4-912 $\delta$ or lys2-1288, whereas the high-copy-number subclones do cause suppression. Therefore, the DNA fragments carried on the class 1 and class 2 clones cause suppression only when present in high copy number. The experiments in this paper focus on results with the class 2 clone.

\section{The class 2 clone contains the SPT11 and SPT12 genes}

We isolated the class 2 clone as a high-copy-number suppressor of $\delta$ insertion mutations. To determine if null mutations in the class 2 locus would cause suppression or some other mutant phenotype, we constructed disruptions at this locus by Tn10-LUK transposon insertion mutagenesis in E. coli (Huisman et al. 1987; see Experimental procedures). Eleven Tn10-LUK insertion mutations that eliminated the class 2 high-copy-number suppression phenotype were recombined into the genome of strain FW1237, replacing the wild-type locus (Rothstein 1983; see Experimental procedures). Haploid strains with these Tnl0-LUK insertions grow more slowly than wild-type strains but are viable, demonstrating that the class 2 locus is not essential for growth. In addition, strains that contain Tn10-LUK disruptions of the class 2 locus exhibit the $\mathrm{Spt}^{-}$phenotype of suppression of $\delta$ insertion mutations. Thus, either overproduction or disruption of the class 2 locus causes suppression of $\delta$ insertion mutations.

Since the class 2 disruption alleles exhibited an $\mathrm{Spt}^{-}$ phenotype, we tested whether the class 2 locus could complement mutations in any previously identified SPT gene. We transformed strains containing representative mutant alleles of the 14 different SPT genes with a lowcopy-number plasmid carrying the class 2 locus. This plasmid complemented the $\mathrm{Spt}^{-}$phenotype of either spt11 or spt12 mutant strains. Mutations in SPT11 and SPT12 are very tightly linked to each other and are suppressors of solo $\delta$ and complete Ty insertion mutations (Fassler and Winston 1988). To prove that the class 2 locus contains the SPT11 and SPT12 genes, we showed that certain class 2 Tn10-LUK disruption alleles fail to complement spt11 and spt12 mutations. Furthermore, all of these mutations segregate in crosses as tightly linked markers. These results demonstrate that the SPT11 and SPT12 genes cause the high-copy-number suppression phenotype of the class 2 clone.

\section{SPT11 and SPT12 are the histone genes HTA1 and HTB1}

We mapped the SPT11 and SPT12 locus to chromosome IV by the $2 \mu$-mapping method (Falco et al. 1982; Falco and Botstein 1983). By tetrad analysis we showed that the SPT11 and SPT12 locus is $12 \mathrm{cM}$ from LYS4 and 16 cM from PET14. From the map distance and the relative frequencies of recombinants between markers in the same cross, we conclude that the map order is CEN4PET14-(SPT11 and SPT12)-LYS4. This is very close to the genetic map position for the histone genes HTA1 and HTB1 (Norris and Osley 1987), suggesting that SPT11 and SPT12 may be these two histone genes. This idea was supported by the observation that the restriction map of the SPT11 and SPT12 locus is identical to that of the HTA1-HTB1 locus (Hereford et al. 1979) with respect to HindIII, PvuII, and BgIII restriction sites. Southern hybridization analysis between a plasmid that contains the HTA1-HTB1 locus and one that contains the SPT11 and SPT12 locus demonstrated that these two loci contain homologous sequences (data not shown).

If SPT11 and SPT12 are HTA1 and HTB1, we would expect that mutations in $H T A 1$ and $H T B 1$ would exhibit 
suppression of $\delta$ insertion mutations. We observed that a deletion of the HTA1-HTB1 locus does exhibit suppression of insertion mutations (strain CC232). To confirm that the SPT11 and SPT12 genes are HTA1 and HTB1 we showed that a deletion of the HTA1-HTB1 locus fails to complement either spt11 or spt12 mutations for suppression.

To verify that the high-copy-number suppression phenotype of the class 2 clone was caused by the HTA1 and HTB1 genes, we examined the effects of 32 Tn10-LUK transposon insertion mutations (see Experimental procedures) in the class 2 clone on suppression of insertion mutations. Eleven of the Tn10-LUK insertions eliminated high-copy-number suppression; all of these insertions mapped within the HTA1-HTB1 region by restriction endonuclease analysis (Fig. 1). The remaining 21 Tn10-LUK insertions that did not affect suppression mapped to regions on either side of the HTA1-HTB1 locus, including the region containing the Protein 1 (PRT1) gene, whose function is unknown (Hereford et al. 1979).

To determine the exact position of five of the Tn10LUK insertion mutations that eliminated high-copynumber suppression, we sequenced their sites of insertion. Two of these insertion mutations are in the coding regions for HTA1 and HTB1: hta1-3: : Tn10-LUK is inserted 27 bp $3^{\prime}$ of the HTA1 translation initiation codon; htb1-5: : Tn10-LUK is inserted 98 bp $3^{\prime}$ of the HTB1 initiation codon. The remaining insertions appear to be located within sequences encoding untranslated portions of the HTA1 and HTB1 transcripts: hta1-1 : :Tn10-LUK and hta1-2: : Tn10-LUK are both inserted 74 bp $3^{\prime}$ of the HTA1 translation stop codon; htb1-4 : : Tn10-LUK is located $4 \mathrm{bp} 5^{\prime}$ of the $H T B 1$ translation start codon. These results prove that high copy number of the HTA1-HTB1 locus causes suppression of $\delta$ insertion mutations. Apparently, overproduction of both $\mathrm{H} 2 \mathrm{~A}$ and $\mathrm{H} 2 \mathrm{~B}$ is necessary to cause suppression, since Tn10-LUK insertions into either HTA1 or HTB1 destroy high-copy-number suppression. Thus, suppression is probably caused by an elevated level of the heterodimer $\mathrm{H} 2 \mathrm{~A}-\mathrm{H} 2 \mathrm{~B}$, since high copy number of either HTA1 or HTB1 alone does not cause suppression.

Increased dosage of other histone loci causes suppression of $\delta$ insertion mutations

The HTA1-HTB1 locus was isolated as a high-copynumber suppressor of $\delta$ insertion mutations; therefore, we tested whether the other histone loci, when in high copy number, would also cause suppression. Saccharomyces cerevisiae has four unlinked histone loci organized as divergently transcribed gene pairs. There are two H2A-H2B gene pairs located at the HTA1-HTB1 and HTA2-HTB2 loci, and two $\mathrm{H} 3-\mathrm{H} 4$ gene pairs encoded by the HHT1-HHF1 and HHT2-HHF2 loci (Hereford et al. 1979; Smith and Murray 1983).

We first tested the high-copy-number HTA2-HTB2 plasmid, pDN242, in strain FW1237 and found that it caused suppression of both his4-912 $\delta$ and $1 y s 2-128 \delta$ from His $^{-}$Lys $^{-}$to His ${ }^{+}$Lys $^{+}$(Table 1). Since the two histone loci HTA1 -HTB1 and HTA2 -HTB2 share some restriction sites and limited homology at the DNA level (Hereford et al. 1979|, we did more detailed restriction mapping and Southern hybridization analysis of the 13 original class 2 clones. We discovered that four of the 13 are actually HTA2 -HTB2 clones. Therefore, both of the $\mathrm{H} 2 \mathrm{~A}-\mathrm{H} 2 \mathrm{~B}$ loci were isolated as high-copy-number suppressors of $\delta$ insertion mutations.

We also tested the high-copy-number plasmids pCC64 and pCC66 containing the H3-H4 loci HHT1-HHF1 and HHT2-HHF2 (Smith and Murray 1983; Smith and Andresson 1983). Both pCC64 and pCC66 suppress his4-9128 from $\mathrm{His}^{-}$to $\mathrm{His}^{+}$but only weakly suppress lys2-128 from Lys ${ }^{-}$to Lys $^{-1+}$ (Table 1). The weak suppression of lys2-128 explains why neither of the $\mathrm{H} 3-\mathrm{H} 4$ loci were isolated in the original screen for strong Lys ${ }^{+}$ transformants. These results demonstrate that increased dosage of any one of the four histone gene pairs causes suppression of $\delta$ insertion mutations (Fig. 2).

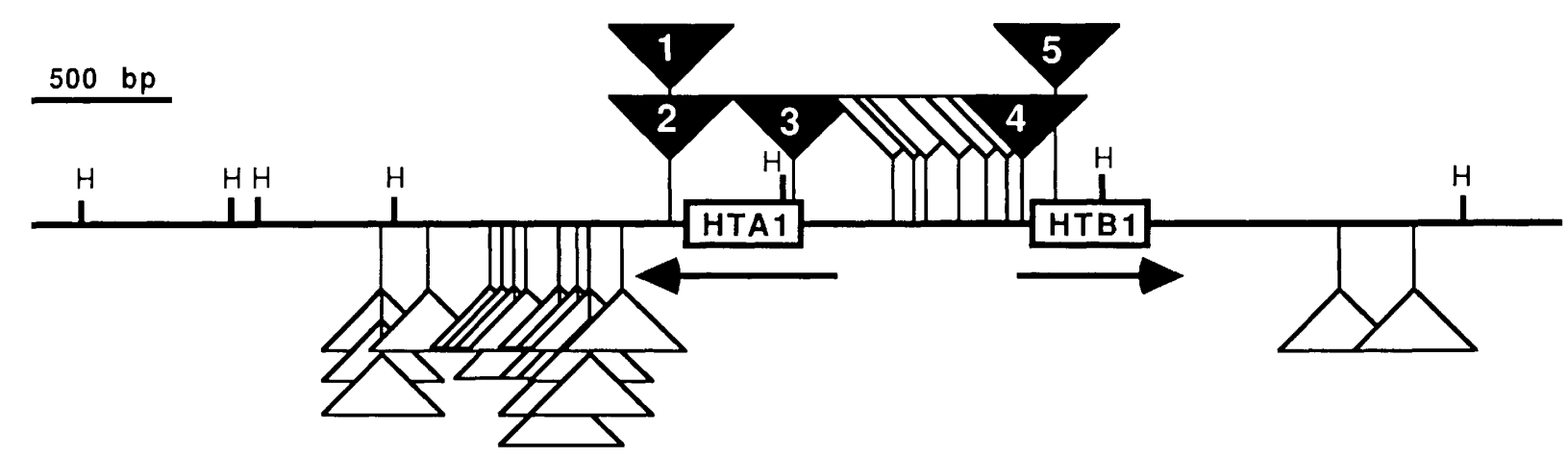

Figure 1. Tn10-LUK insertions at the HTA1-HTB1 locus. Each triangle represents a single 6.09-kb Tn10-LUK element. Triangles below the line indicate Tn10-LUK insertions that did not affect the high-copy-number suppression phenotype of plasmid pCC61. Triangles above the line represent Tn10-LUK insertions that eliminated the high-copy-number suppression phenotype of pCC61. The 11 insertions shown above the line were transplaced into the genome as described in Experimental procedures. The black triangles represent Tn10-LUK elements whose sites of insertion were sequenced. The black arrows indicate the approximate positions of the HTA1 and HTB1 transcripts. (H) HindIII restriction sites. 
Table 1. Suppression of insertion mutations by histone genes in high copy number

\begin{tabular}{|c|c|c|c|}
\hline Plasmid & $\begin{array}{l}\text { Histone locus } \\
\text { in high } \\
\text { copy number }\end{array}$ & his $4-912 \delta$ & $1 y s 2-128 \delta$ \\
\hline YEp24 & none & - & - \\
\hline pCC61 & HTA1-НTB1 & + & + \\
\hline pDN242 & HTA2-HTB2 & + & + \\
\hline pCC64 & $H H T 1-H H F 1$ & + & $-1+$ \\
\hline pCC66 & HHT2-HHF2 & + & $-1+$ \\
\hline pCC67 & HTA1-HTB1, HHT1-HHF1 & - & - \\
\hline
\end{tabular}

Symbols indicate growth on media lacking histidine for his4$912 \delta$ and on media lacking lysine for lys2-128ס. (+) Strong growth; $|-1+|$ weak growth; $|-|$ no growth. Suppression was determined by replica plating onto SD plates supplemented with the appropriate amino acids.

\section{Overproducing all four histones eliminates suppression}

We have demonstrated that increased dosage of histone gene pairs that encode either an $\mathrm{H} 2 \mathrm{~A}-\mathrm{H} 2 \mathrm{~B}$ or an $\mathrm{H} 3-\mathrm{H} 4$ dimer set causes suppression of $\delta$ insertion mutations. To test whether suppression is due to the creation of an imbalance between dimer sets, we constructed a highcopy-number plasmid, pCC67, which contains all four histone genes from the HTA1-HTB1 and HHT1-HHF1 loci. This plasmid does not confer suppression when transformed into strain FW1237; the strain remains His $^{-}$and Lys ${ }^{-}$(Table 1; Fig. 2). The HTB1 and HHT1 transcript levels in this transformant are overproduced to approximately the same degree (two- to fivefold) as in strains that contain high-copy-number clones of the individual HTA1-HTB1 or HHT1-HHF1 loci (approximately threefold overproduction; Fig. 3). We conclude that altering the ratio of $\mathrm{H} 2 \mathrm{~A}-\mathrm{H} 2 \mathrm{~B}$ to $\mathrm{H} 3-\mathrm{H} 4$ dimers causes suppression of $\delta$ insertion mutations. This result is similar to that of Meeks-Wagner and Hartwell (1986), whose experiments suggested that the ratio of $\mathrm{H} 2 \mathrm{~A}$ and
$\mathrm{H} 2 \mathrm{~B}$ to $\mathrm{H} 3$ and $\mathrm{H} 4$ is important for the normal fidelity of mitotic chromosome transmission.

\section{Reduced histone gene dosage causes suppression}

We next examined the effect of reduced histone gene dosage on suppression of $\delta$ insertion mutations for all four histone loci. Since each histone locus is duplicated, any gene disruption will halve the gene dosage (the level of histone protein may not decrease twofold if the different loci are not expressed at the same level or if the remaining gene can compensate for the reduced gene dosage|. We have demonstrated that deletion of the HTA1-HTB1 locus causes suppression of $\delta$ insertion mutations and that Tn10-LUK insertion mutations in either HTA1 or HTB1 also cause suppression (Table 2).

While deletion of HTA1-HTB1 causes suppression, deletion of HTA2-HTB2 does not cause suppression (Table 2). One explanation for this result is the observation that the HTA1-HTB1 locus can increase its expression to compensate for the loss of HTA2-HTB2, but that HTA2$H T B 2$ is unable to compensate for the loss of $H T A 1$ HTB1 (Norris and Osley 1987). Alternatively, suppression of $\delta$ insertion mutations caused by $\Delta(h t a 1-h t b 1)$ could be due to the loss of specific $\mathrm{H} 2 \mathrm{~A}$ and $\mathrm{H} 2 \mathrm{~B}$ protein subtypes encoded at the HTA1-HTB1 locus (Wallis et al. 1980; Choe et al. 1982). To distinguish between these possibilities, we duplicated the HTA2-HTB2 locus in a $\Delta(h t a 1-h t b 1)$ strain. Duplication of HTA2-HTB2 eliminates the suppression phenotype of $\Delta(h t a 1-h t b 1)$ (Table 2 ). This result demonstrates that suppression of $\delta$ insertion mutations caused by deletion of the HTA1-HTB1 locus is due to a reduction in $\mathrm{H} 2 \mathrm{~A}$ and $\mathrm{H} 2 \mathrm{~B}$ levels. Most of the phenotypes Norris and Osley (1987) observed as a result of deleting HTA1-HTB1 were also due to a reduction in $\mathrm{H} 2 \mathrm{~A}$ and $\mathrm{H} 2 \mathrm{~B}$ levels.

Deletion of either of the H3-H4 loci does not cause

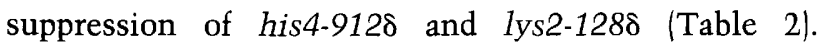
Perhaps each $\mathrm{H} 3-\mathrm{H} 4$ locus can compensate for loss of the other, so that deletion of one locus does not result in a reduction in the level of $\mathrm{H} 3$ and $\mathrm{H} 4$ proteins. Alternatively, a reduction in $\mathrm{H} 3-\mathrm{H} 4$ levels may not cause suppression of $\delta$ insertion mutations.

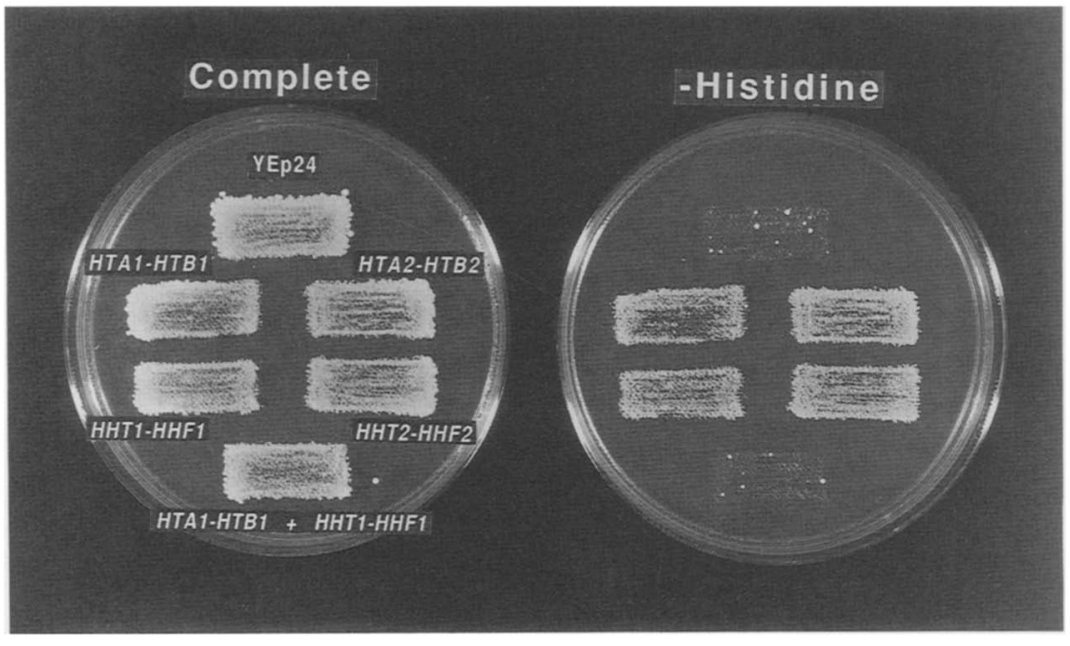

Figure 2. Suppression of his4-912 $\delta$ by highcopy-number histone genes. Strain FW1237 was transformed with high-copy-number plasmids carrying the histone loci indicated in the figure: YEp24; pCC61(HTA1-HTB1); pDN242(HTA2-HTB2); pCC64(HHT1-HHF1); pCC66(HHT2-HHF2); pCC67|HTA1-HTB1 and HHT1-HHF1). The transformants were grown in patches on an SD plate supplemented with all amino acid requirements and then replicaplated onto media lacking histidine (-Histidinel and to media containing all amino acid requirements (Complete). 


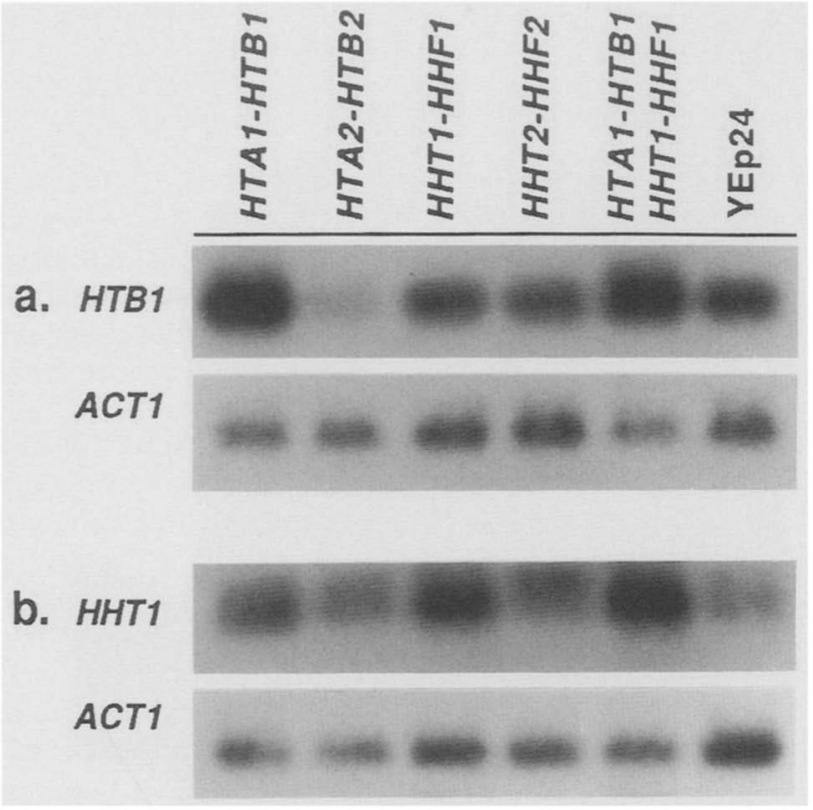

Figure 3. Northern analysis of histone RNA in strains with histone genes in high copy number. Strain FW1237 was transformed with high-copy-number plasmids carrying the histone loci indicated in the figure: pCC61(HTA1-HTB1) pDN242(HTA2-HTB2); pCC64(HHT1-HHF1); pCC66)HHT2HHF2); pCC67(HTA1-HTB1 and HHT1-HHF1); YEp24. Total RNA was isolated from the transformants. (a) Four micrograms of RNA was run per lane. The probes were an HTB1-specific fragment and an $A C T 1$-specific fragment. $H T B 1$ mRNA is approximately $0.6 \mathrm{~kb}$ in length; $A C T 1$ mRNA is $1.4 \mathrm{~kb}$. (The amount of HTB1 transcript is reduced four- to fivefold in the strain containing pDN242 HTA2-HTB2) relative to the amount in the strain containing YEp24, probably reflecting dosage compensation by the HTA1-HTB1 locus.) (b) One microgram of RNA was run per lane. The probes were an $H H T 1$-specific fragment and an $A C T 1$-specific fragment. HHT1 mRNA is approximately $0.7 \mathrm{~kb}$ long.

\section{Deletion of HTAl and HTBl alters transcription of

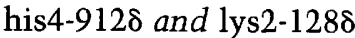

In all cases studied, spt mutations suppress insertion mutations at the transcriptional level (Silverman and Fink 1984; Winston et al. 1984a; Clark-Adams and Winston 1987; Fassler and Winston 1988). Northern hybridization analysis shows that suppression of his $4-912 \delta$ and lys2-128 by $\Delta(h t a 1-h t b 1)$ also occurs via specific transcriptional alterations (Fig. 4). Wild-type HIS4 and LYS2 transcription appear unaffected by $\Delta(h t a 1-h t b 1)$.

In his4-912 $\delta$ strains, HIS4 transcription occurs in both HTA1-HTB1 and $\Delta($ hta1-htb1) genetic backgrounds, but the predominant transcripts differ in size. In an HTA1HTB1 his4-9128 strain, which is His $^{-}$, the most abundant HIS4 transcript initiates in the $\delta$ sequence and is nonfunctional for HIS4 expression (Silverman and Fink 1984) (Fig. 4a, lane 3). In the $\Delta(h t a 1-h t b 1)$ his $4-912 \delta$ strain, which is $\mathrm{His}^{+}$, two transcripts are apparent. A wild-type-size HIS4 transcript is present in addition to a reduced amount of the $\delta$-initiated transcript (Fig. 4a, lane 4). Thus, $\Delta(h t a 1-h t b 1)$ restores a functional HIS4 transcript to his4-9128, apparently by altering the initiation site of the HIS4 mRNA. We have observed similar transcriptional alterations at his4-912 $\delta$ caused by highcopy-number clones containing any one of the four histone loci (data not shown).

For lys2-1288, a different type of transcriptional alteration was observed. In contrast to his4-912 $\delta$, at which the $\delta$ sequence is inserted in the $5^{\prime}$ noncoding region of $H I S 4$, the $\delta$ sequence at lys2-128 $\delta$ is inserted within the $5^{\prime}$ end of the LYS2 coding region (Clark-Adams and Winston 1987). We used two LYS2 probes to study transcription of lys2-1288: pFW47, an internal LYS2 probe, and pFW112, a probe containing 5 ' noncoding and coding sequences (see Experimental procedures). These probes were used individually in initial experiments and were mixed for later experiments. For an HTA1-HTB1 lys2-1288 strain, which is Lys $^{-}$, only one LYS2 transcript is present: a 580-bp transcript, detected with the $5^{\prime}$ probe pFW112 (Fig. 4b, lane 3; Clark-Adams and Winston 1987). The size of this transcript is consistent with that of a transcript that initiates at the normal LYS2 transcription start site and terminates in the $\delta$ sequence. No major LYS2 mRNA is detected with the internal LYS2 probe. In a $\Delta(h t a 1-h t b 1)$ lys2-128 double mutant (Fig. 4b, lane 4), which is $\mathrm{Lys}^{+}$, two transcripts are present. The $5^{\prime}$ LYS2 probe detects a short transcript similar in size to the $5^{\prime}$ transcript seen in the HTA1HTB1 lys2-1288 strain. In addition, the internal LYS2 probe detects a new LYS2 transcript that is slightly shorter than the wild-type LYS2 mRNA. We believe this new transcript is functional since the strain is $\mathrm{Lys}^{+}$. Apparently, the $\Delta(h t a 1-h t b 1)$ mutation alters transcription at lys2-128 to create a functional LYS2 transcript. (In both HTA1-HTB1 and $\Delta$ (hta1-htb1) backgrounds, the internal LYS2 probe sometimes detects a minor LYS2 transcript of approximately $3.8 \mathrm{~kb}$ ).

\section{Discussion}

Our results demonstrate that changes in the dosage of the histone locus HTA1-HTB1 can alter transcription in

Table 2. Suppression of insertion mutations by mutations in histone loci

\begin{tabular}{|c|c|c|}
\hline Relevant genotype & his4-9128 & lys2-1288 \\
\hline Wild type & - & - \\
\hline hta1-3::Tn10-LUK & + & + \\
\hline$h t b 1-5:: T n 10-L U K$ & + & + \\
\hline$\Delta(h t a 1-h t b 1)$ & + & + \\
\hline$\Delta(h t a 2-h t b 2)$ & - & - \\
\hline \multicolumn{3}{|l|}{$\Delta(h t a 1-h t b 1)$} \\
\hline HTA2-HTB2/HTA2-HTB2 & - & - \\
\hline$\Delta(h h t 1-h h f 1)$ & - & - \\
\hline$\Delta(h h t 2-h h f 2)$ & - & - \\
\hline
\end{tabular}

Symbols indicate growth on media lacking histidine for his4$912 \delta$ and on media lacking lysine for lys2-128ס. (+) Strong growth $;(-)$ no growth. Suppression was determined by replica plating onto SD plates supplemented with the appropriate amino acids. HTA2-HTB2/HTA2-HTB2 indicates a duplication of this locus. 


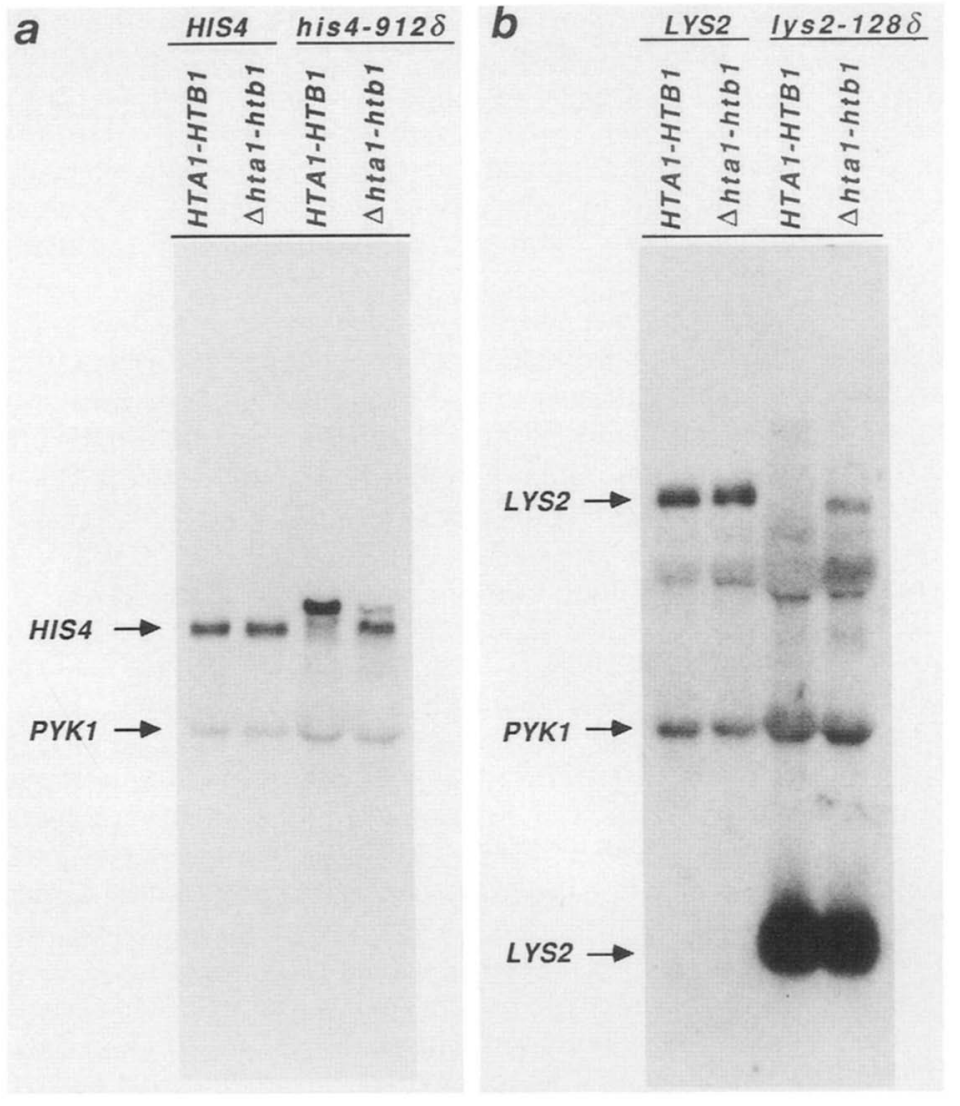

Figure 4. Northern analysis of HIS4- and LYS2-specific RNA in HTA1-HTB1 and $\Delta(h t a 1-h t b 1)$ strains. Total RNA was isolated from the strains indicated. One microgram of RNA was run in lanes 1 and 2; $5 \mu \mathrm{g}$ was run in lanes 3 and 4 . The strains represented are (left to right): S288C, DN106, FW1237, and CC232. (a) The probes were pFW45(HIS4) and pFR2(PYK1). HIS4 mRNA is $2.6 \mathrm{~kb}$ long. $(b)$ The probes were pFW47(internal LYS2), pFW112(5' LYS2), and pFR2. LYS2 mRNA is $4.2 \mathrm{~kb}$. The faint band present in all four lanes is caused by the presence of $25 \mathrm{~S}$ rRNA.
S. cerevisiae. The cases for which we have observed transcriptional changes are solo $\delta$ insertion mutations at the HIS 4 and LYS2 genes. The transcriptional alterations result in suppression of these insertion mutations, changing the phenotypes they confer from $\mathrm{His}^{-}$and Lys $^{-}$to His ${ }^{+}$and Lys ${ }^{+}$. The suppression phenotype has allowed us to score the effects of both amplification and mutation of all four $S$. cerevisiae histone loci on transcription. Our analysis suggests that it is the alteration of the proper stoichiometry of histone dimer sets that causes the transcriptional changes.

Two results directly suggest that an imbalance of histone dimer sets, rather than an imbalance of individual histone proteins or a change in the absolute level of histones, is responsible for the effects we have observed. First, both histones $\mathrm{H} 2 \mathrm{~A}$ and $\mathrm{H} 2 \mathrm{~B}$ must be overproduced to cause suppression of insertion mutations; a mutation in either HTA1 or HTB1 on a high-copy-number plasmid abolishes the suppression phenotype. Second, highcopy-number plasmids that contain any one of the histone gene pairs cause suppression of solo $\delta$ insertion mutations, yet when two different loci encoding all four histones are on the same high-copy-number plasmid, no suppression is observed.

While high copy number of any of the four histone loci causes suppression of insertion mutations, deletion of only one particular histone locus, HTA1-HTB1, causes suppression. At this locus, either a deletion encompassing both genes or mutations within either gene alone cause suppression. Suppression is observed for the
Tn10-LUK insertion mutations described in this work and for the spontaneous HTA1 and HTB1 mutations isolated as spt11 and spt12 suppressor mutations (Fassler and Winston 1988). This result is consistent with the model that the level of histone dimers is the critical parameter causing a transcriptional change; either a deletion of HTA1-HTB1 or a single mutation in one of the two genes should reduce the level of $\mathrm{H} 2 \mathrm{~A}-\mathrm{H} 2 \mathrm{~B}$ dimers.

A deletion of the other H2A-H2B locus, HTA2-HTB2, does not cause suppression of insertion mutations; this is probably due to dosage compensation by HTA1-HTB1 in strains deleted for HTA2-HTB2 (Norris and Osley 1987). In such strains, the level of expression of HTA1HTB1 is increased, resulting in near-wild-type levels for these two histones. By this hypothesis, it is the level of $\mathrm{H} 2 \mathrm{~A}-\mathrm{H} 2 \mathrm{~B}$ dimers, rather than an alteration in the relative levels of a particular $\mathrm{H} 2 \mathrm{~A}$ or $\mathrm{H} 2 \mathrm{~B}$ subtype (Wallis et al. 1980; Choe et al. 1982), that is responsible for the suppression phenotype. This model is supported strongly by our observation that a strain containing an HTA1-HTB1 deletion and a duplication of HTA2-HTB2 does not suppress insertion mutations.

Deletion of either of the H3-H4 loci, HHT1-HHF1 or HHT2-HHF2, also does not cause suppression. One explanation is that these loci may both dosage compensate for each other, as does HTA1-HTB1 for HTA2-HTB2. If this were true, then neither deletion would lower the level of $\mathrm{H} 3$ and $\mathrm{H} 4$ enough to cause a suppression phenotype. Alternatively, it is possible that a reduction of $\mathrm{H} 3-\mathrm{H} 4$ dimers does not lead to the same alteration in 
transcription as does a reduction in $\mathrm{H} 2 \mathrm{~A}-\mathrm{H} 2 \mathrm{~B}$ dimers. The latter possibility is more difficult to reconcile with the result that a high-copy-number plasmid that contains either HHT1-HHF1 or HHT2-HHF2 causes suppression.

Deletion of the HTA1-HTB1 locus causes a variety of mutant phenotypes (Norris and Osley 1987). Therefore, it is conceivable that the transcriptional changes we have observed are indirect, caused by some other effect of altered histone gene dosage. One possibility is that the transcriptional alterations are occuring via the heat shock response, which is expressed constitutively in strains deleted for HTA1-HTB1 (Norris and Osley 1987). If transcription from $\delta$ sequences were reduced under heat shock conditions, then the $\delta$ insertion mutations could be suppressed in the $\Delta(h t a 1-h t b 1)$ strain because the normally strong $\delta$ promoters are reduced in efficiency, allowing weaker promoters at HIS4 and LYS2 to compete for transcription factors. However, we have observed no change in the level of Ty transcripts during heat shock as measured by Northern hybridization analysis (D. Norris and H. Iida, unpubl.; Clark-Adams and Winston, unpubl.).

A plausible explanation for the transcriptional alterations we have observed is that changing the level of histone dimer sets either reduces the number of nucleosomes or alters nucleosomal structure, thereby causing a perturbation in chromatin structure that allows different transcription initiation sites to be utilized. Lorch et al. (1987) demonstrated that, in vitro, transcription cannot initiate in a nucleosome. Perhaps the insertion mutations we have studied change nucleosome phasing such that nucleosomes block transcription initiation sites at HIS4 and LYS2. When the number of nucleosomes is reduced or nucleosomal structure is altered, an initiation site for a functional transcript would now be accessible to the cell's transcription machinery. Micrococcal nuclease and DNase I digestion studies will help to identify changes in chromatin structure at his4-912 $\delta$ and 1ys2-128 caused by altering histone gene dosage.

Either overproduction or underproduction of histone dimer sets can cause transcriptional changes. There is evidence that an equimolar ratio of $\mathrm{H} 2 \mathrm{~A}-\mathrm{H} 2 \mathrm{~B}$ to $\mathrm{H} 3-\mathrm{H} 4$ is necessary for proper chromatin formation in vitro $/ \mathrm{Ca}$ merini-Otero et al. 1976). An increase or decrease in the level of either dimer set would upset this ratio, and, as discussed by Meeks-Wagner and Hartwell (1986), might promote the assembly of aberrant nucleosomal structures and/or reduce the number of nucleosomes. The level of functional complexes involved in other processes, including bacteriophage morphogenesis (Floor 1970; Sternberg 1976) and bacterial chemotaxis (DeFranco and Koshland 1981) has been shown to be sensitive to altered stoichiometry.

The transcriptional alterations we have observed as the result of changing histone gene dosage occur at insertion mutations at the HIS4 and LYS2 genes. We did not see any alteration in transcription of the wild-type HIS4, LYS2, and PYK1 genes. Likewise, Han et al. (1987) did not see any effect on transcription of the $S$. cerevi- siae CUP1 gene under conditions in which no histone $\mathrm{H} 2 \mathrm{~B}$ is being synthesized. The level of expression of wild-type genes may be unaffected by the degree to which we have perturbed histone levels. Alternatively, transcriptional effects caused by altered histone levels may be detected only under certain conditions such as mutation of a promoter or absence of a trans-acting factor.

The original purpose of the screen for high-copynumber suppressors was to isolate new genes that shared phenotypes with the SPT6 gene. Either too many or too few copies of the SPT6 gene cause suppression of $\delta$ insertion mutations (Clark-Adams and Winston 1987). We isolated the HTA1-HTB1 locus as a high-copynumber suppressor and subsequently demonstrated that mutations in the locus also cause suppression of $\delta$ insertion mutations. In addition, the transcriptional alterations at his4-912 $\delta$ and $1 y s 2-128 \delta$ observed in the $\Delta(h t a 1-h t b 1)$ strains are similar to those seen in spt6 mutant strains (Clark-Adams and Winston 1987). Whether the function of the SPT6 gene product is related to histone function will be addressed in future experiments.

In conclusion, we have demonstrated that alterations in histone gene dosage can cause specific transcriptional changes in yeast. Suppression of insertion mutations, the system by which we have detected these changes, is sensitive to small variations in levels of gene expression that are easy to detect. We anticipate that this system will provide a powerful genetic tool to assay for changes in chromatin structure caused by mutations in histone genes as well as by mutations in other genes whose products are important for chromatin structure.

\section{Experimental procedures}

Yeast strains and genetic methods

The $S$. cerevisiae strains constructed for these studies are de rived from strain S288C and are listed in Table 3. We have used standard genetic nomenclature for the histone genes. Uppercase letters denote the dominant (wild-type) allele and lowercase letters denote the recessive (mutant) allele. The $\Delta(h t a 1-h t b 1)$ and $\Delta(h t a 2-h t b 2)$ alleles are described in Norris and Osley (1987). The $\Delta(h h t 1-h h f 1)$ and $\Delta(h h t 2-h h f 2)$ alleles were provided by $M$. Mitchell Smith. Standard methods for mating, sporulation, and tetrad analysis were used (Mortimer and Hawthrone 1969; Sherman et al. 1978). All media were made as described by Sherman et al. (1978).

\section{Transformations}

Yeast cells were transformed by the lithium acetate method (Ito et al. 1983). Escherichia coli HB101 (Boyer and Roulland-Dussoix 1969) was transformed to ampicillin resistance as decribed by Maniatis et al. (1982).

\section{Enzymes}

Restriction enzymes and T4 DNA ligase were purchased from New England Biolabs, Inc., and used as suggested by the supplier. 
Table 3. Yeast strains

\begin{tabular}{|c|c|}
\hline Strain & Genotype \\
\hline S288C & MATa ga12 hap1 \\
\hline FW1237 & 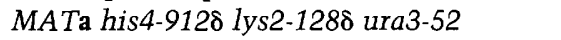 \\
\hline FW1238 & 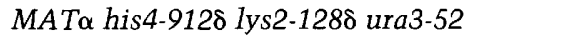 \\
\hline $\mathrm{CC} 232$ & $\begin{array}{l}\text { MATa } \Delta(h t a 1-h t b 1) \text { his } 4-912 \delta \text { lys2-128ס } \\
\quad \text { ura3 }\end{array}$ \\
\hline $\mathrm{CC} 217$ & $\begin{array}{l}\text { MAT } \alpha(\text { hta2-htb2) his } 4-912 \delta \text { lys2-128ס } \\
\text { ura3-52 }\end{array}$ \\
\hline CC249 & MATa $\Delta(h h t 1-h h f 1)$ his4-9128 lys2 ura3-52 \\
\hline $\mathrm{CC} 251$ & MATa $\Delta(h h t 2-h h f 2)$ his4-9128 lys2 ura3-52 \\
\hline $\mathrm{CC} 204$ & 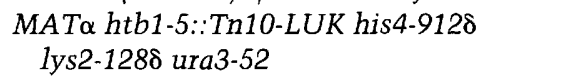 \\
\hline $\mathrm{CC} 240$ & 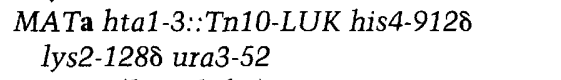 \\
\hline CC260 & $\begin{array}{l}\text { MATa } \Delta(\text { hta1-htb1) HTA2-HTB2/HTA2- } \\
\text { HTB2 his3 lys2-128ס ura3 }\end{array}$ \\
\hline DN106 & MATa $\Delta($ hta1-htb1) his3 ura3 \\
\hline $\mathrm{CC} 131$ & 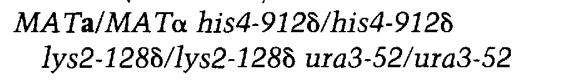 \\
\hline
\end{tabular}

\section{DNA preparation and analysis}

Yeast DNA minipreps for recovery of plasmids from $10-\mathrm{ml}$ cultures were done as described by Winston et al. (1983). E. coli plasmid mini-preps were prepared by the alkaline lysis method (Birnboim and Doly 1979). DNA restriction fragments were analyzed by electrophoresis through $0.6 \%$ Seakem ME agarose gels that were run in $0.5 \times \mathrm{E}$ buffer (Roeder and Fink 1980). We purified restriction fragments from agarose gels by electroelution. Southern blot hybridization analysis of yeast genomic DNA was performed as described (Roeder and Fink 1980). ${ }^{32} \mathrm{P}$ Labeled DNA probes were prepared by nick-translation (Rigby et al. 1977).

\section{Plasmids}

Plasmid vectors used for subcloning were YCp50 (Johnston and Davis 1984), YEp24 (Botstein et al. 1979), YIp5 (Parent et al. 1985), and pCGS42 (provided by Collaborative Research). Highcopy-number plasmids of histone genes were constructed as described below and are diagrammed in Figure 5. A 6-kb Sall restriction fragment containing the HTA1-HTB1 locus was cloned into the SalI site of YEp24 to create pCC61. In addition to HTA1 and HTB1, the Sall fragment in pCC61 contains a gene of unknown function, PRT1 (Protein 1) (Hereford et al. 1979). Plasmid pDN242 contains a 3.55-kb EcoRI-HindIII fragment from the HTA2-HTB2 locus cloned into the EcoRI and BamHI sites of YIp5 and a 2.24-kb EcoRI fragment from $2 \mu$ circle cloned into the EcoRI site of YIp5. Plasmid pCC64 contains a $6.7-\mathrm{kb}$ HindIII fragment containing the HHT1-HHF1 locus (provided by M. Mitchell Smith) cloned into the HindIII site of YEp24. In addition to HHT1 and HHF1, the insert in pCC64 contains SMT1, a gene of unknown function (Smith and Murray 1983). Plasmid pCC66 contains two HindIII fragments, $2.6 \mathrm{~kb}$ and $1.48 \mathrm{~kb}$, from the HHT2-HHF2 locus (provided by $\mathrm{M}$. Mitchell Smith) cloned into the HindIII site of pCGS42. Plasmid pCC67 contains four histone genes (HTA1, HTB1, $H H T 1$, and HHF1) and was constructed by cloning a $6.4-\mathrm{kb}$ HTA1-HTB1 BamHI fragment and a 5.4-kb HHT1-HHF1 BamHI-HindIII fragment into the HindIII and BamHI sites of pCGS42.

\section{Hybridization probes}

Plasmids and DNA fragments used as probes were as follows: for HIS4, pFW45, a HIS4 internal BgIII-Sall restriction fragment in pBR322 (Winston et al. 1984a); for LYS2, pFW47, a LYS2 internal BglII-XhoI restriction fragment in pBR322, and pFW112, a LYS2 EcoRI-BgIII fragment spanning the 5' end of LYS2, cloned into pBR322 (Clark-Adams and Winston 1987); for PYK1, pFR2, a clone containing the PYK1 gene (provided by Pratima Sinha); for $A C T 1$, a 3.2-kb BamHI-EcoRI restriction fragment containing the $A C T 1$ gene (derived from plasmid pRB149 provided by David Botstein); for HTB1, a 1.3-kb HindIII restriction fragment containing the $3^{\prime}$ of the HTB1 gene; for HHT1, a 3.5-kb HindIII-SmaI restriction fragment containing the $H H T 1$ gene and the $S M T 1$ gene.

\section{RNA blot hybridization analysis}

Cells for RNA isolation were grown in supplemented SD medium to $1 \times 10^{7}$ cells $/ \mathrm{ml}$, and yeast RNA isolated as described (Carlson and Botstein 1982). For RNA blots and hybrdization analysis, we followed the instructions for GeneScreen (New England Nuclear) using the dextran sulfate method. RNA was cross-linked onto GeneScreen by exposure to UV radiation (1200 $\mu \mathrm{W} / \mathrm{cm}^{2}$ for $2 \mathrm{~min}$ ) (Church and Gilbert 1984). The amount of RNA per lane was standardized by hybridization to pFR2 or to a restriction fragment containing the ACT1 gene. Bands on autoradiograms were quantitated by densitometry on an LKB Bromma Ultroscan XL Laser Densitometer.

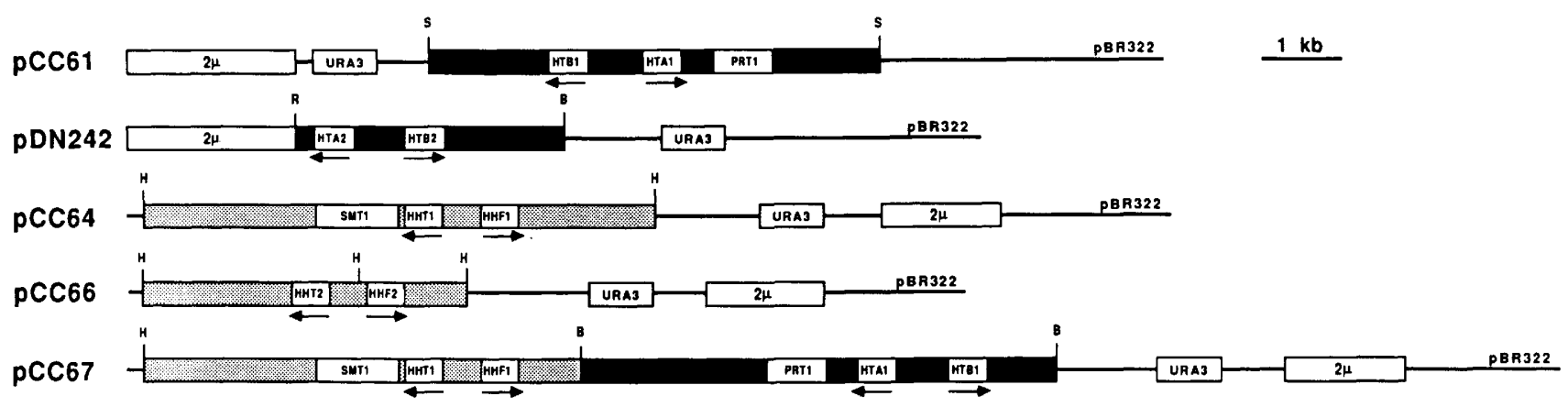

Figure 5. High-copy-number plasmids containing the histone loci. The autonomously replicating plasmids diagrammed all contain the $2 \mu$ origin of replication and the URA3 gene as a selectable marker in yeast. Each plasmid is shown with the EcoRI site of pBR322 located at the far left end. The plasmids were constructed as described in Experimental procedures. Only relevant restriction sites are listed. (S) SaII; (R) EcoRI; (B) BamHI; (H) HindIII. 


\section{Construction and analysis of HTA1-HTB1 null alleles}

A Sall restriction fragment containing the HTA1-HTB1 locus was subcloned into YEp24. The resulting subclone, pCC61, was subjected to Tn10-LUK transposon insertion mutagenesis (Huisman et al. 1987). The Tnl0-LUK element carries the URA3 gene as a selectable marker in yeast. Derivatives of pCC61 that contain individual Tn10-LUK insertions were transformed into strain FW1237 and $\mathrm{Ura}^{+}$transformants were screened for the ability to suppress $\delta$ insertion mutations. The positions of the Tn10-LUK insertions were determined by restriction mapping. Eleven Tn10-LUK insertions within the HTA1-HTB1 locus which eliminate the suppression phenotype of pCC61 were transplaced into the yeast genome as follows: SalI restriction fragments containing the HTA1-HTB1 locus disrupted by a given Tn10-LUK insertion were purified and used to transform yeast by selection for Ura ${ }^{+}$. A double crossover event will replace one of the genomic HTA1-HTB1 locus alleles with the Tn10-LUK-disrupted HTA1-HTB1 allele (Orr-Weaver et al. 1981; Rothstein 1983). Two of the Tn10-LUK insertions were transformed into the diploid strain CC131. To analyze the phenotype of the disruption in haploid cells, we sporulated the $\mathrm{Ura}^{+}$transformants and dissected tetrads. The remaining nine Tn10-LUK insertions in the HTA1-HTB1 locus were transplaced directly into haploid strain FW1237. Southern blot hybrdization analysis of the Ura ${ }^{+}$haploid transformants confirmed that all eleven Tnl0-LUK insertions were integrated at the HTA1-HTB1 locus.

\section{DNA sequence analysis}

The positions of the Tn10-LUK transposon insertion mutations at the HTA1-HTB1 locus were determined by DNA sequence analysis, using a primer complementary to the end of the Tn10-LUK element: $5^{\prime}$ d(CAAGATGTGTATCCACC)- $3^{\prime}$ (made by Paul Tempst with an Applied Biosystems 380B DNA Synthesizer). Plasmids containing the Tnl0-LUK insertions were digested with restriction enzymes to produce a restriction fragment with one end inside the Tn10-LUK element and the other end within the HTA1-HTB1 locus. These restriction fragments were isolated by electroelution, denatured, annealed to primer, and sequenced using a modification of the Sanger technique as described by Bartlett et al. (1986). $\left[\alpha^{-35} S\right]$ dATP was purchased from New England Nuclear.

\section{Acknowledgments}

We thank George Church and Elizabeth Malone for a critical reading of the manuscript. We are indebted to $M$. Mitchell Smith for providing deletions of HHT1-HHF1 and HHT2-HHF2. This work was supported by National Institutes of Health grant GM32967, National Science Foundation grant DCB8451649, and grants from Stroh Brewery Company and Monsanto Company to F.W. J.S.F. was supported by National Institutes of Health postdoctoral fellowship IF32 GM10168 and a Charles A. King Trust fellowship from the Medical Foundation.

\section{References}

Bartlett, J.A., R.K. Gaillard, Jr., and W.K. Joklik. 1986. Sequencing of supercoiled plasmid DNA. Biotechniques 4: $208-210$.

Birnboim, H.C. and J. Doly. 1979. A rapid alkaline extraction procedure for screening recombinant plasmid DNA. Nucleic Acids Res. 7: 1513-1523.

Botstein, D., S.C. Falco, S.E. Stewart, M. Brennan, S. Scherer, D.T. Stinchcomb, K. Struhl, and R.W. Davis. 1979. Sterile host yeasts (SHY): A eukaryotic system of biological containment for recombinant DNA experiments. Gene 8: 17-24.

Boyer, H.W. and D. Roulland-Dussoix. 1969. A complementation analysis of the restriction and modification of DNA in E. coli. J. Mol. Biol. 41: 458-472.

Camerini-Otero, R.D., B. Sollner-Webb, and G. Felsenfeld. 1976. The organization of histones and DNA in chromatin: Evidence for an arginine-rich histone kernel. Cell 8: 333347.

Carlson, M. and D. Botstein. 1982. Two differentially regulated mRNAs with different 5 ' ends encode secreted and intracellular forms of yeast invertase. Cell 28: 145-154.

Choe, J., D. Kolodrubetz, and M. Grunstein. 1982. The two yeast histone H2A genes encode similar protein subtypes. Proc. Natl. Acad. Sci. 79: 1484-1487.

Church, G.M. and W. Gilbert. 1984. Genomic sequencing. Proc. Natl. Acad. Sci. 81: 1991-1995.

Clark-Adams, C.D. and F. Winston. 1987. The SPT6 gene is essential for growth and is required for $\delta$-mediated transcription in Saccharomyces cerevisiae Mol. Cell. Biol. 39: 679686.

DeFranco, A.L. and D.E. Koshland, Jr. 1981. Molecular cloning of chemotaxis genes and overproduction of gene products in the bacterial sensing system. I. Bacteriol. 147: 390-400.

Falco, S.C. and D. Botstein. 1983. A rapid chromosome-mapping method for cloned fragments of yeast DNA. Genetics 105: 857-872.

Falco, S.C., Y. Li, J.R. Broach, and D. Botstein. 1982. Genetic properties of chromosomally integrated $2 \mu$ plasmid DNA in yeast. Cell 29: 573-584.

Fassler, J.S. and F. Winston. 1988. Isolation and analysis of a novel class of suppressor of Ty insertion mutations in Saccharomyces cerevisiae. Genetics 118:203-212.

Floor, E. 1970. Interaction of morphogenetic genes of bacteriophage T4. J. Mol. Biol. 47: 293-306.

Han, M., M. Chang, U. Kim, and M. Grunstein. 1987. Histone $\mathrm{H} 2 \mathrm{~B}$ repression causes cell-cycle-specific arrest in yeast: Effects on chromosomal segregation, replication, and transcription. Cell 48: 589-597.

Hereford, L., K. Fahmer, J. Woolford, Jr., M. Rosbash, and D.B. Kaback. 1979. Isolation of yeast histone genes H2A and H2B. Cell 18: 1261-1271.

Huisman, O., W. Raymond, K. Froehlich, P. Errada, N. Kleckner, D. Botstein, and M.A. Hoyt. 1987. A Tn10-lacZkanR-URA3 gene fusion transposon for insertion mutagenesis and fusion analysis of yeast and bacterial genes. $\mathrm{Ge}$ netics 116: 191-199.

Ito, H., Y. Fukuda, K. Murata, and A. Kimura. 1983. Transformation of intact yeast cells treated with alkali cations. $J$. Bacteriol. 153: 163-168.

Johnston, M. and R.W. Davis. 1984. Sequences that regulate the divergent GAL1-GAL10 promoter in Saccharomyces cerevisiae. Mol. Cell. Biol. 4: 1440-1448.

Lorch, Y., J.W. LaPointe, and R.D. Kornberg. 1987. Nucleosomes inhibit the initiation of transcription but allow chain elongation with the displacement of histones. Cell 49: 203210.

Maniatis, T., E.F. Fritsch, and J. Sambrook. 1982. Molecular cloning: A laboratory manual. Cold Spring Harbor Laboratory, Cold Spring Harbor, New York.

Mathis, D., P. Oudet, and P. Chambon. 1980. Structure of transcribing chromatin. Prog. Nucl. Acad. Res. Mol. Biol. 24: $1-55$.

Meeks-Wagner, D. and L.H. Hartwell. 1986. Normal stoichiometry of histone dimer sets is necessary for high fidelity of 
mitotic chromosome transmission. Cell 44: 53-63.

Moore, G.D., D.A. Sinclair, and T.A. Grigliatti. 1983. Histone gene multiplicity and position effect variegation in Drosophila melanogaster Genetics 105: 327-344.

Mortimer, R.K. and D.C. Hawthorne. 1969. Yeast genetics. In The yeasts, (ed. A.H. Rose and J.S. Harrison), vol. 1, pp. 385-460. New York. Academic Press.

Nasmyth, K.A. 1982. The regulation of yeast mating-type chromatin structure by $S I R$ : An action at a distance affecting both transcription and transposition. Cell 30: 567-578.

Neigeborn, L., J.L. Celenza, and M. Carlson. 1987. SSN20 is an essential gene with mutant alleles that suppress defects in SUC2 transcription in Saccharomyces cerevisiae. Mol. Cell. Biol. 7: 672-678.

Norris, D. and M.A. Osley. 1987. The two gene pairs encoding $\mathrm{H} 2 \mathrm{~A}$ and $\mathrm{H} 2 \mathrm{~B}$ play different roles in the Saccharomyces cerevisiae life cycle. Mol. Cell. Biol. 7:3473-3481.

Orr-Weaver, T.L., J.W. Szostak, and R.J. Rothstein. 1981. Yeast transformation: a model system for the study of recombination. Proc. Natl. Acad. Sci. 78: 6354-6358.

Parent, S.A., C.M. Fenimore, and K.A. Bostian. 1985. Vector systems for the expression, analysis and cloning of DNA sequences in $S$. cerevisiae. Yeast 1: 83-138.

Pederson, D.S., F. Thoma, and R.T. Simpson. 1986. Core particle, fiber, and transcriptionally active chromatin structure. Annu. Rev. Cell. Biol. 2: 117-147.

Rigby, P.W.J., M. Dieckmann, C. Rhodes, and P. Berg. 1977. Labeling deoxyribonucleic acid to high specfiic activity in vitro by nick translation with DNA polymerase I. $J$. Mol. Biol. 113: 237-251.

Roeder, G.S. and G.R. Fink. 1980. DNA rearrangements associated with a transposable element in yeast. Cell 21: 239249.

Rothstein, R.J. 1983. One-step gene disruption in yeast. Methods Enzymol. 101: 202-211.

Sherman, F., G.R. Fink, and C.W. Lawrence. 1978. Methods in yeast genetics, rev. ed. Cold Spring Harbor Laboratory, Cold Spring Harbor, New York.

Silverman, S.J. and G.R. Fink. 1984. Effects of Ty insertions on HIS4 transcription in Saccharomyces cerevisiae. Mol. Cell. Biol. 4: 1246-1251.

Smith, M.M. and O.S. Andresson. 1983. DNA sequences of yeast $\mathrm{H} 3$ and $\mathrm{H} 4$ histone genes from two non-allelic gene sets encode identical $\mathrm{H} 3$ and $\mathrm{H} 4$ proteins. $/$. Mol. Biol. 169: 663-690.

Smith, M.M. and K. Murray. 1983. Yeast H3 and H4 histone messenger RNAs are transcribed from two non-allelic gene sets. J. Mol. Biol. 169: 641-661.

Sternberg, N. 1976. A genetic analysis of bacteriophage $\lambda$ head assembly. Virology 71: 568-582.

Wallis, J.W., L. Hereford, and M. Grunstein. 1980. Histone H2B genes of yeast encode two different proteins. Cell 22: 799805.

Winston, F., F. Chumley, and G.R. Fink. 1983. Eviction and transplacement of mutant genes in yeast. Methods Enzymol. 101: 211-228.

Winston, F., K.J. Durbin, and G.R. Fink. 1984a. The SPT3 gene is required for normal transcription of Ty elements in $S$. cerevisiae. Cell 39: 675-682.

Winston, F., D.T. Chaleff, B. Valent, and G.R. Fink. 1984b. Mutations affecting Ty-mediated expression of the HIS4 gene of Saccharomyces cerevisiae. Genetics 107: 179-197.

Winston, F., C. Dollard, E.A. Malone, J. Clare, J.G. Kapakos, P. Farabaugh, and P.L. Minehart. 1987. Three genes are required for trans-activation of Ty transcription in yeast. $\mathrm{Ge}$ netics 115: 649-656. 


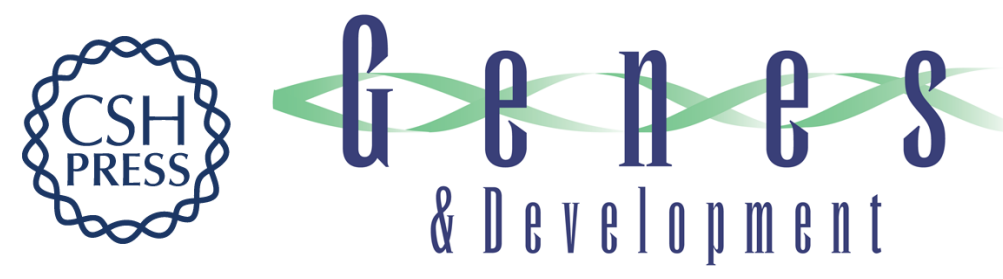

\section{Changes in histone gene dosage alter transcription in yeast.}

C D Clark-Adams, D Norris, M A Osley, et al.

Genes Dev. 1988, 2:

Access the most recent version at doi:10.1101/gad.2.2.150

References This article cites 41 articles, 15 of which can be accessed free at: http://genesdev.cshlp.org/content/2/2/150.full.html\#ref-list-1

License

Email Alerting Receive free email alerts when new articles cite this article - sign up in the box at the top Service right corner of the article or click here.

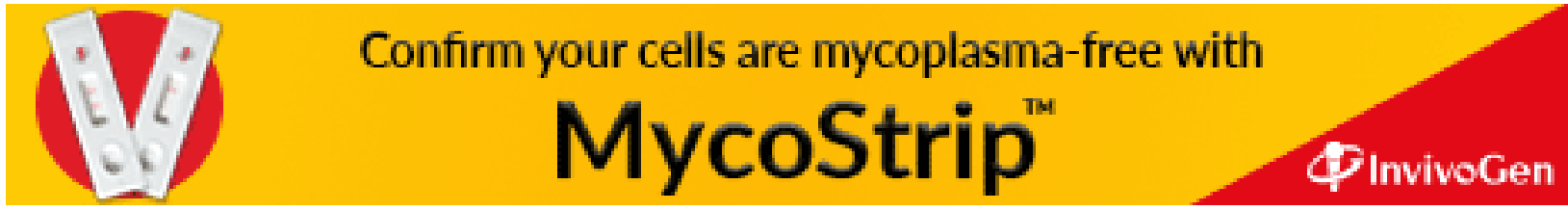

